\title{
Tracking in Molecular Bioimaging
}

\author{
Erik Meijering ${ }^{1}$ Ihor Smal ${ }^{1}$ Gaudenz Danuser ${ }^{2}$ \\ ${ }^{1}$ Biomedical Imaging Group Rotterdam, Erasmus MC—University \\ Medical Center Rotterdam, Rotterdam, The Netherlands \\ ${ }^{2}$ Laboratory for Computational Cell Biology, Department of Cell Biology, \\ The Scripps Research Institute, La Jolla, CA, USA
}

IEEE Signal Processing Magazine, vol. 23, no. 3, May 2006, pp. 46-53

A utomated tracking and analysis of moving objects in image sequences has been and continues to be one of the major themes in digital image analysis research. This is not surprising in view of its many applications in video surveillance, multimedia services, automated vehicle guidance and driver assistance, remote sensing and meteorology, and medical imaging. It is also a recurring theme in molecular biology. By their very nature, biomolecular systems are dynamic, and it is one of the major challenges of biomedical research and pharmaceutical industries in the postgenomic era to unravel the spatial and temporal relationships of these complex systems and to devise strategies to manipulate them. Results in this area can be expected to have profound social and economic impact in the near future, as they can be harnessed to improve human health and well-being [1]. Studies into biomolecular dynamics generate ever increasing amounts of image data. To be able to handle these data and to fully exploit them for describing biological processes on a quantitative level and building accurate mathematical models of dynamic structures, computerized motion analysis is rapidly becoming a requisite [2].

Over the past decades, a number of image analysis techniques have been developed in support of such studies, the details of which were often buried in the small print of the methods sections of papers published in the biology and biophysics literature. The majority of these techniques were based on rather rudimentary principles, however. The purpose of this article is to stimulate the application of more advanced computer vision techniques to tracking in biological molecular imaging, by surveying the literature and sketching the current state of affairs in the field for a signal and image processing audience. After describing the basic principles of visualizing molecular dynamics in living cells and giving some examples of biological molecular dynamics studies, we summarize the problems and limitations intrinsic to imaging at this scale. Then we discuss the main ingredients of the commonly used tracking paradigm and subsequently reconsider its competence by comparing it to certain aspects of visual motion perception in human beings, keeping in mind the complexity and variability of biological image data. We conclude by summarizing the main points of attention for future research and the challenges that lie ahead. 


\section{STUDYING BIOLOGICAL MOLECULAR DYNAMICS}

Currently the most important imaging tool for studying dynamic processes in living cells still is light microscopy [3]. The use of light microscopes for biological experimentation and investigation goes back to Antoni van Leeuwenhoek (1632-1723), who discovered the motion of bacteria, sperm cells, blood cells and more, using a simple magnifying lens. It is only since relatively recently, however, that light microscopy became mature enough to allow in vivo imaging of molecular complexes and even single molecules (see also Vonesch et al., this issue). Apart from substantial improvements in optics hardware and the development of increasingly sensitive electronic imaging sensors, a key factor was the discovery, cloning, and expression of the jellyfish green fluorescent protein (GFP). This enabled visible fluorescence to be encoded into a specific gene of interest, which, in turn, enables one to tag and optically detect virtually any protein of interest in living cells. In recent years, many GFP variants have been developed with different spectral properties, enabling simultaneous detection of differently labeled proteins and studying their interaction [4].

Combined with time-lapse imaging, these developments have provided powerful tools to study the dynamic properties and functions of proteins. An example of this is fluorescence recovery after photobleaching (FRAP). With this technique, the fluorescence in a region of interest in the cell is selectively quenched by intense illumination. Subsequent analysis of the degree and rate of fluorescence recovery in this region allows to determine kinetic parameters (diffusion coefficients, mobile fraction, binding/dissociation rates) of the labeled protein [3]. A complementary example is fluorescence loss in photobleaching (FLIP), in which a region is repeatedly bleached while the loss of fluorescence in other regions is monitored. This allows to study the continuity of regions within cells, as only connected regions will show a loss of fluorescence. While these techniques are useful for determining ensemble average parameters, more detailed studies into the different modes of motion (immobile, directed, confined, tethered, normal or anomalous diffusion) of subpopulations of intracellular components require single-particle tracking [5] (the subject matter of this article), which aims at tracking and motion analysis of individual particles.

A few examples of biological molecular dynamic processes and images (see Figure 1) acquired for studies into these phenomena may help one to appreciate the challenges of tracking. One of the intracellular structures being investigated intensively in biology is the cytoskeleton, which consists of several subsystems of densely interwoven and highly plastic networks of filamentous polymers. One category of cytoskeleton polymers is constituted by the microtubules. These are required for a variety of cellular functions and their dynamic behavior is regulated by many factors. Insight into these processes are obtained, for example, from studies using GFP fused to proteins that associate with the distal ends of growing microtubules [6]. Another largely unresolved mystery being actively researched is the molecular machinery involved in DNA repair and replication. It consists of a collection of protein complexes that act in a sequential but highly coordinated fashion [7]. Quantitative motion analyses may unveil how exactly this cast of proteins interacts. Continuing with DNA-associated phenomena, we mention the study of telomere dynamics. Telomeres are the nucleoprotein complexes at the natural ends of chromosomes, which distinguish them from DNA breaks which activate repair mechanisms, and together with specialized binding proteins are crucial for maintaining chromosome stability, topology, and integrity [8]. Many of the molecular mechanisms involved still remain to be discovered. As a final example we mention the study of androgen receptors [9], proteins generally found in the cytoplasm that specifically bind androgens to form complexes that migrate to the nucleus to induce the transcription of specific segments of DNA. The transcriptional activity, subnuclear distribution, and nuclear mobility of these complexes are under continuing investigation.

These and numerous other studies generate vast amounts of image data, where the dynamics of hundreds or thousands of particles need to be analyzed quantitatively and as completely as possible to reveal functional behavior and to detect rare events suggestive of functional heterogeneity. This may lead to the for- 

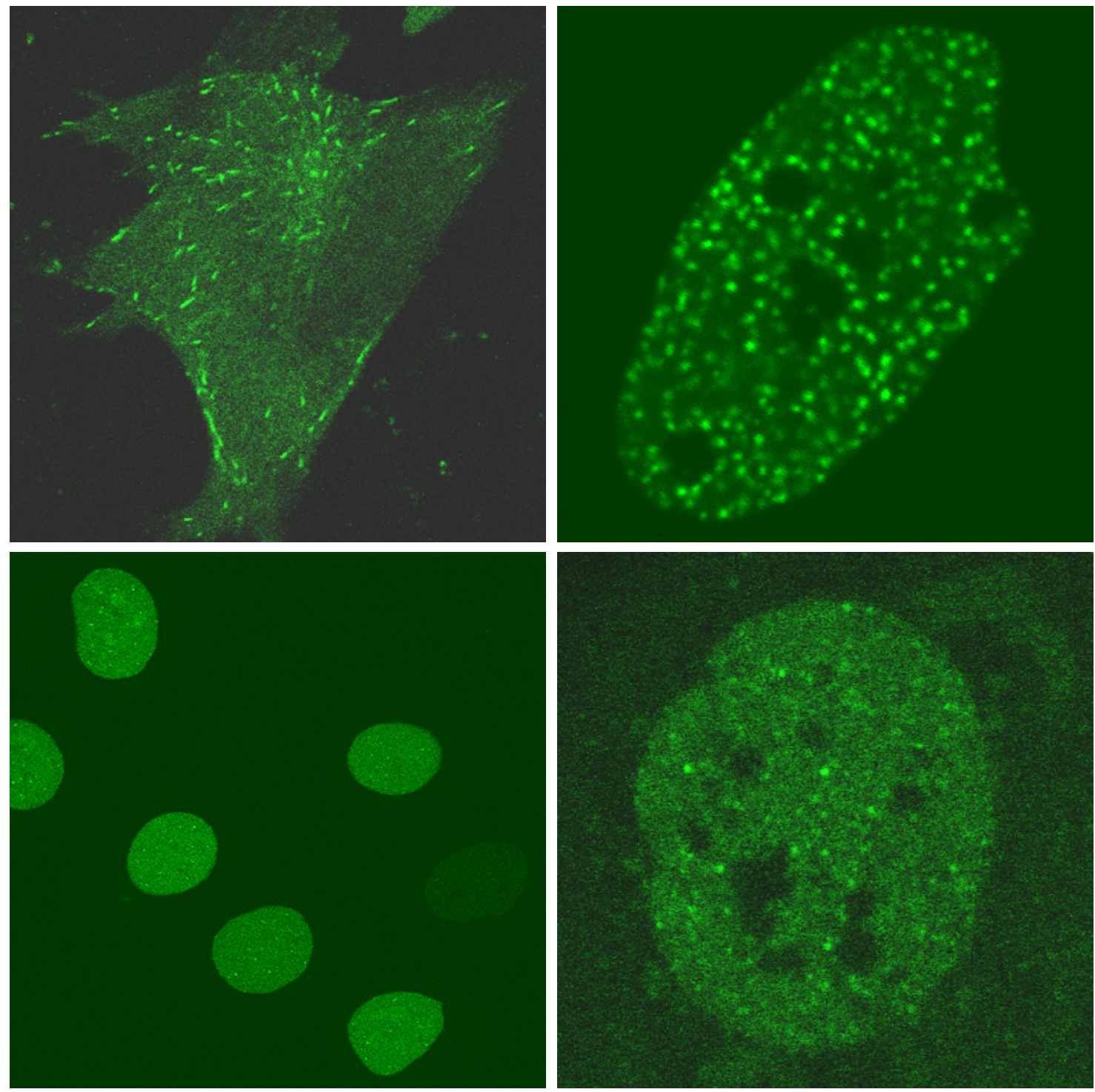

FIGURE 1 Examples of images acquired for different biological molecular dynamics studies based on GFP labeling and fluorescence microscopy. Top left: Plus-ends of microtubules in a mouse embryonic fibroblast cell (single frame, $\pm 65 \times 65 \mu \mathrm{m}$, from a $2 \mathrm{D}$ time-lapse study, 2 seconds between frames). Top right: Proliferating cell nuclear antigen (PCNA) in the nucleus of a Chinese hamster ovary cell (single frame, $\pm 25 \times 25 \mu \mathrm{m}$, from a 2D time-lapse study, 30 seconds between frames). Bottom left: Telomeres in the nuclei of several HeLa cells (single plane, $\pm 0.9 \times 0.9 \mathrm{~mm}$, from a single stack, 10 planes per stack, from a 3D time-lapse study, 10 minutes between stacks). Bottom right: Androgen receptors in the nucleus of a Hep3B cell (single frame, $\pm 30 \times 30 \mu \mathrm{m}$, from a 2D time-lapse study, about 6 seconds between frames). See the acknowledgements at the end of this article for credits.

mulation of comprehensive models of molecular processes. Obviously, manual tracking is labor intensive, costly, inaccurate, and poorly reproducible, and usually only a small fraction of the data can be analyzed in this manner. This calls for renewed efforts in developing computational image analysis tools. 


\section{FUNDAMENTAL PROBLEMS AND LIMITATIONS}

Achieving robustness and high accuracy in tracking and motion analysis in images obtained by light microscopy is hampered by three factors. The first is the limited spatial resolution of the microscope. Even an optimally designed and employed microscope, which to a good approximation can be modeled as a linear shift-invariant system with a finite point-spread function (PSF), suffers from diffraction. The Fraunhoferdiffraction limited PSF (normalized to unit magnitude at the origin) of a confocal microscope with circular aperture and operating under design conditions is given by [10]

$$
\operatorname{PSF}(r, z)=\left|\int_{0}^{1} 2 J_{0}(\alpha r \rho) \exp \left(-2 i \gamma z \rho^{2}\right) \rho d \rho\right|^{2}
$$

in which

$$
\alpha=\frac{2 \pi \mathrm{NA}}{\lambda} \text { and } \gamma=\frac{\pi \mathrm{NA}^{2}}{2 \lambda}
$$

and where $r=\sqrt{x^{2}+y^{2}}$ denotes radial distance to the optical axis, $z$ is the axial distance to the focal plane, $i$ the imaginary unit number, $J_{0}$ the zeroth-order Bessel function of the first kind, NA the numerical aperture of the objective lens, and $\lambda$ the wavelength of the light emitted by the specimen. This function (see also Figure 2) is band-limited, in both the lateral (in-plane) and the axial (across-plane) direction, with radial cut-off frequencies of $\omega_{r}=2 \alpha$ and $\omega_{z}=2 \gamma$, respectively. Although the blurring due to the PSF can be reduced afterwards to some degree by deconvolution (see Sarder and Nehorai, this issue), it rules out morphological analysis of individual molecules and impedes studying their interaction.

The second confounding factor is noise. Even if all sources of noise due to system imperfections are reduced to a minimum, the signal-to-noise ratio (SNR) is still limited because of the randomness introduced by the quantum nature of light. This randomness follows a Poisson distribution and is therefore not independent of the signal. Moreover, in most experiments the signal is kept to a minimum, since high illumination rapidly quenches fluorescence and may disrupt the cellular and molecular processes being studied. And for the very same reason, the number of images taken is usually minimized as well. As a result, both the SNR (see again Figure 1) and the temporal resolution are usually quite low.

A third complicating factor is the large variability of biological image data. Foremost this has to be attributed to the intrinsic heterogeneity of molecular systems. In addition, a lack of standardization in the acquisition protocols among studies may result in imagery of the same molecular process with quite different appearance and quality. The quality of images may not even be constant within one experiment, for example because of a degradation of the fluorescent probes over time (photobleaching). This is in sharp contrast with medical investigations, where routine clinical studies are based on standardized imaging protocols, leading to more consistent image quality. All these factors put high demands on the design of automated image analysis techniques. This applies not only to tracking and motion analysis, but to other biological image analysis problems as well (see Vonesch et al., this issue).

\section{COMMON TRACKING PARADIGM}

Computational image analysis tools for (semi-)automated tracking of single molecules or molecular compounds within living cells have been developed and reported since the early 1980s. The basic concepts underlying the vast majority of published methods are virtually the same, however. The commonly used approach to motion tracking consists of at least the following steps (see also Figure 3): preprocessing the image data, detecting individual particles per time point, linking particles detected at successive time points, and analyzing the results. We discuss the state of the art for each of these steps. 

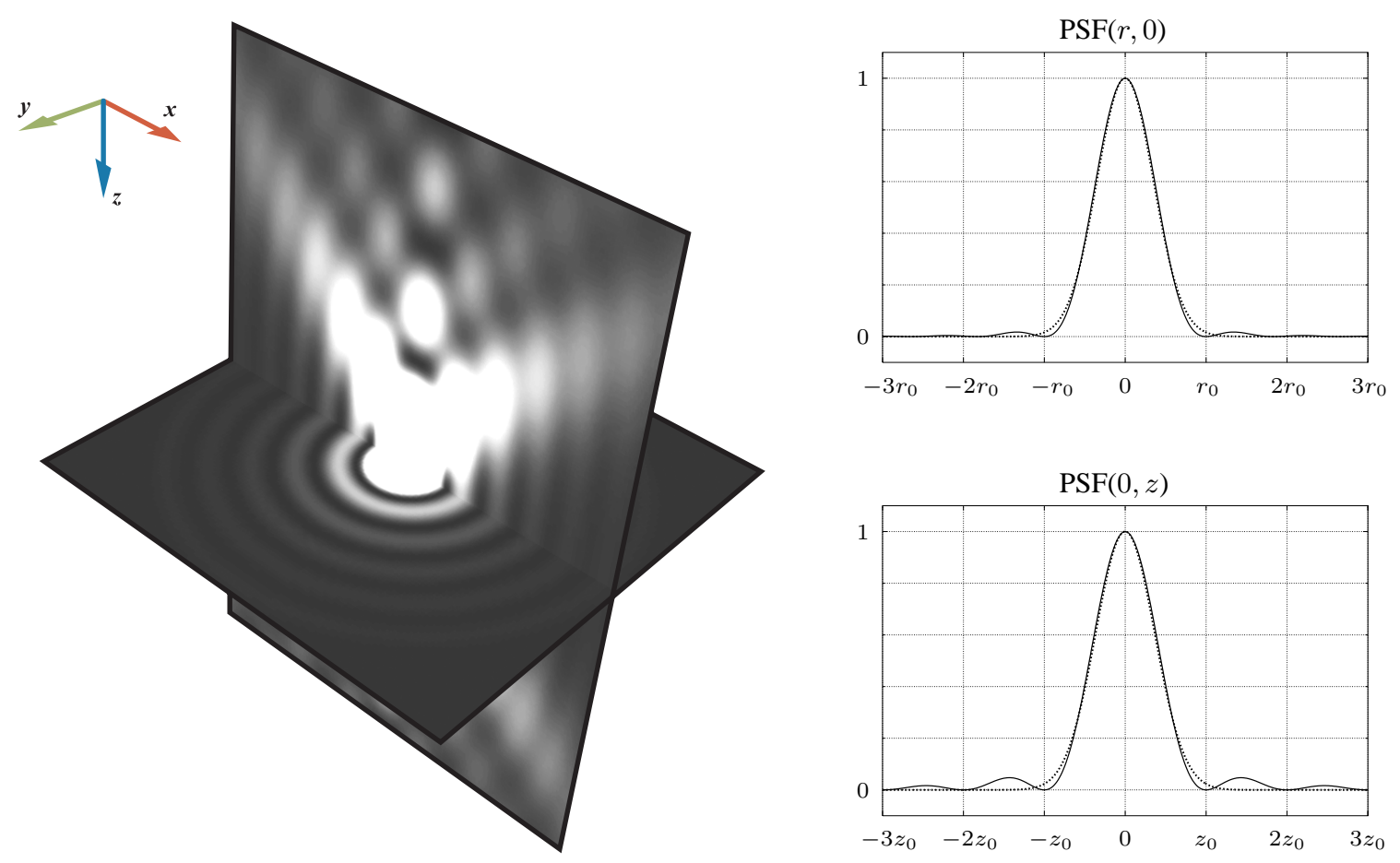

FIGURE 2 Left: Cross-sectional view of the theoretical PSF (strongly contrast-enhanced to show its ringing structure) of a confocal microscope operating under optimal conditions. Right: The PSF (solid lines) and its least-squares Gaussian approximation (dotted lines) in both the lateral (top plot) and axial (bottom plot) direction. The standard deviations are $\sigma_{r}=0.349 r_{0}$ and $\sigma_{z}=0.365 z_{0}$, respectively, with $r_{0}$ and $z_{0}$ being the first roots of the PSF in the respective directions.

\section{PREPROCESSING THE IMAGE DATA}

One of the most important factors influencing tracking algorithm performance is the SNR. It has been demonstrated by experiments on artificial data [11] that the accuracy of commonly used tracking algorithms degrades rapidly as the SNR drops below $20 \mathrm{~dB}$ and becomes unacceptable below $12 \mathrm{~dB}$. (Note that due to the signal dependence of the noise, different definitions of SNR exist, depending on how the noise is measured. Here we define the noise level as the standard deviation of the intensities within the object, not the background.) Such levels are, however, not uncommon in fluorescence imaging. Moreover, it has been shown [12] that on short time scales, localization inaccuracies caused by noise in the images may make particle diffusion processes appear anomalous even if they are normal. It is therefore of crucial importance to enhance the SNR for subsequent particle tracking by applying noise reduction techniques. Since the most dominant noise source possesses Poisson rather than Gaussian characteristics, nonlinear filtering techniques are frequently used for this purpose. Examples range from simple median filtering [13] to more sophisticated anisotropic nonlinear diffusion filtering techniques [14,15].

Quantitative motion analysis of particles within a living cell obviously requires that the global motion of the cell itself is known as well [2]. Clearly, tracking is considerably easier when cells retain their position and morphology throughout an image sequence. If this condition cannot be imposed at the time of image acquisition, one may attempt to enforce it retrospectively. One approach to accomplish this is to apply (nonrigid) image registration, a vigorously studied problem in medical imaging, receiving more and more 


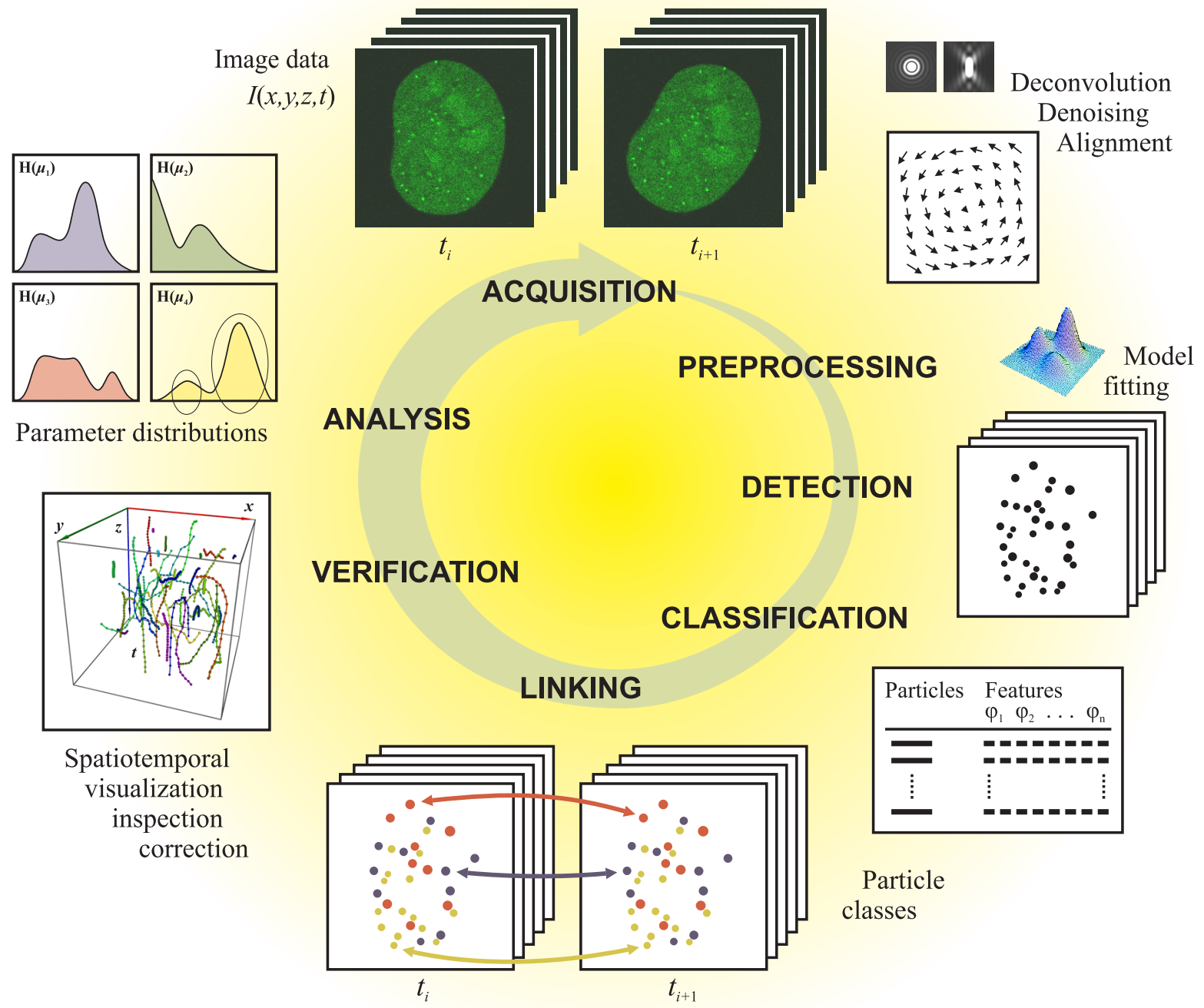

FIGURE 3 Schematic of the steps commonly involved in molecular dynamics studies. Depending on the type of particles and parameters studied, image data is acquired in either 2D, 3D (the case shown), or even 4D (multi-channel 3D) over time. Preprocessing of the raw data, such as deconvolution, noise reduction, and spatial alignment of the successive images, is usually required to considerably improve tracking results. Detection of relevant particles in the images is most effectively done by fitting a predefined model to the data. Once detected, a host of features can be computed for each particle, which may serve to divide particles into classes, if applicable. Feature values are also required for computing correspondence probabilities in the subsequent linking step. The resulting particle tracks may be verified and, if necessary, corrected using efficient spatiotemporal representations. Finally, a variety of dynamics parameters may be computed from the tracks. Ultimately these should lead to new insights, which, in turn, will stimulate the acquisition of new data, as alluded to by the circular structure of the drawing.

attention also in biological imaging [16]. This is done either by maximizing the similarity of the graylevel distributions of successive images in the sequence according to some predefined criterion [13] or by computing global position and orientation features derived from the distributions [17]. Another approach is 
to explicitly segment and track the boundaries of the cells over time. Here, the matching may be performed by iterative closest point algorithms or by optimizing deformable models (more information on tracking at the cellular level can be found in the article by Zimmer et al., this issue).

\section{DETECTING SUBRESOLUTION PARTICLES}

In order to have an idea of the type of motion analysis studies that can be carried out using light microscopy, it is important to know the level of accuracy that can be attained in pinpointing single molecules. The resolution of a microscope is inversely proportional to the cut-off frequency of the optical transfer function (OTF), which for high-NA imaging (using oil immersion lenses) amounts to about 200nm laterally and 600nm axially. Compared to the size of proteins (on the order of nanometers) this is rather poor and prohibits studying morphologic properties and dynamic phenomena within a fluorescent spot. The location of the corresponding subresolution particle, however, can be determined to much greater accuracy. When considering optical factors only, the fundamental limit of the localization accuracy is approximately given by [18]

$$
\epsilon=\frac{\sigma}{\sqrt{N}}
$$

where $\sigma$ denotes the standard deviation of a Gaussian approximation of the PSF in any spatial direction and $N$ the number of detected photons coming from the particle during the time period over which fluorescent signal is integrated. For practical values of the parameters involved, this amounts to an accuracy in the order of a few nanometers. When including the effects of spatial discretization (sampling), and noise sources other than the quantum nature of light, the localization error may increase to a few tens of nanometers. These numbers, which have been confirmed by empirical studies [11], are at least one order of magnitude below the extension of the PSF and thus the resolution power for separating two objects.

A number of fundamentally different approaches exist to estimating the positions of particles from individual images of a series. Most particle tracking algorithms published to date are based on either one or a combination of these. The computationally simplest approach is to calculate the centroids, or centers of (intensity) mass, of relevant spots. This requires segmentation of the image to suppress irrelevant background structures, usually done by thresholding based on intensity or other image features. Another, computationally more demanding approach is local image registration, where for each spot in one image the local intensity distribution serves as a template to be matched with neighboring distributions in the next image. This requires choosing and optimizing a similarity measure, for which normalized cross-correlation or the sum of (squared) intensity differences are often used. A conceptually somewhat similar but still distinct approach is to fit a predefined mathematical model of the spot intensity distribution. Usually this comes down to least-squares fitting of a Gaussian approximation of the PSF.

These approaches were quantitatively compared under different controlled conditions using artificial 2D time-lapse image sequences [11]. It was demonstrated that for subresolution particles, Gaussian fitting is the best method by several criteria, which intuitively is explained by the fact that in this context it constitutes the (near-)matched filtering approach to detection. Given the superiority of this approach, attempts have been made to extend it to simultaneous detection and localization of multiple particles separated by less than the PSF extension (appearing as overlapping spots in the images), using Gaussian mixture models. These consist of a linear combination of kernels, the number and parameters of which are optimized simultaneously (in either a bottom-up or a top-down fashion) according to some goodness-of-fit criterion. It has been demonstrated experimentally [19] that such methods are indeed capable of separating spots significantly below the inverse of the OTF cut-off frequency. Importantly, with this approach, the resolution is no longer dependent primarily on the PSF extension, but is essentially determined by the SNR of the image data. Resolving particles under these circumstances means how high a localization accuracy can be achieved to 
decide whether the coordinate difference between two particles is statistically significant. As shown by the cited studies, Gaussian fitting breaks down at an SNR of about 12dB. Other studies [20] seem to indicate that multiscale approaches are potentially more robust against noise.

\section{LINKING DETECTED PARTICLES}

Once particles have been detected in all relevant frames of a sequence, a correspondence between them needs to be established. In most practical situations this is by no means trivial, as the number of detected particles will generally not be constant over time. Limitations in the image acquisition process may cause not all particles to be captured and resolved at all times: particles may enter or exit the field of view, they may approach each other to distances that are no longer resolvable so that they merge into a single spot, or, conversely, a spot that seemed to represent a single particle in one frame may turn out be a cluster of particles splitting off in the next. In addition, limited detector performance at low SNRs almost certainly leads to varying degrees of under- or oversegmentation (depending on the parameter settings). In combination, these factors seriously complicate the development of linking strategies.

As in any correspondence problem, the linking of particles detected in successive frames requires the specification of a distance measure, where 'distance' may be defined in terms of spatial proximity as well as similarity in appearance or conformity to expectation. The latter types of features may be computed from the image data, such as size (volume or surface area), integrated intensity or intensity curvature, or they may be derived from the evolving trajectory, such as velocity (magnitude as well as orientation) or the difference in extrapolated position [14]. Together with spatial proximity, these features may be combined into a single distance measure either deterministically or by using fuzzy-logic based approaches [2]. In some applications, where it is known a priori that each particle belongs to only one of a finite number of categories, some of these features may be used in a classification procedure which first separates particles into biologically meaningful subsets [19], to be kept separated in the linking stage.

Methods for linking corresponding particles in successive frames can roughly be classified into "local" and "global". The former type of methods operate in a per-particle fashion: each particle in one frame is linked to a particle in the next (or previous) frame that minimizes a predefined distance measure. (Often this involves specifying a maximum allowable distance, indicating track initiation or termination.) This is the most frequently used approach to linking and may yield satisfactory results in scenes with relatively low particle densities and well-separated spots. In more complex situations with much higher densities and overlapping spots, the linking problem cannot be solved unambiguously without involving neighboring or even all detected particles and finding the optimal correspondences for them simultaneously. Global correspondence search strategies are well-known in image processing [21]. However, many of these perform poorly when applied to biological data because of too simplistic (mostly global) assumptions of particle motion modes, which cannot cope with the intrinsic heterogeneity of motion within one particle trajectory as well as among particles. In addition, these strategies are computationally more demanding.

\section{ANALYZING TRACKING RESULTS}

Before embarking on a thorough quantitative analysis of the results of fully automated tracking algorithms, it is good practice to first examine and verify these qualitatively. Especially at low SNRs, detection and linking errors may easily occur, even with current state-of-the-art algorithms, and require manual correction afterwards. Simply browsing through the data in a frame-by-frame fashion, as was done in early studies, is cumbersome and does not provide sufficient insight into the interrelations between detected features. In the past couple of years, more effective ways to represent and visualize spatiotemporal data have appeared in the literature (see Figure 4 for examples), based on (combined) volume and surface rendering techniques [2]. 

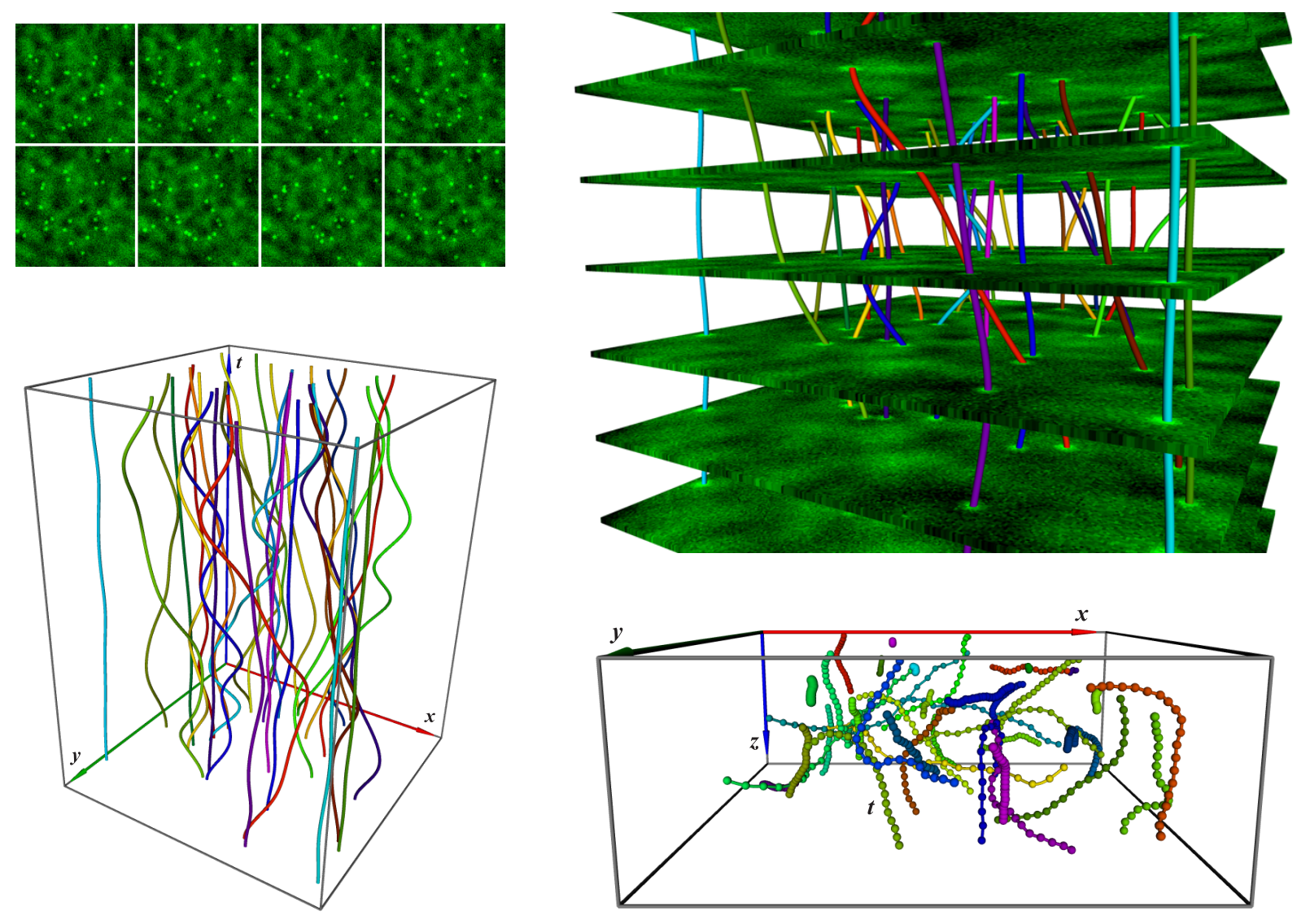

FIGURE 4 Different visualizations of time-lapse image data and particle tracks using representations similar to [14]. Left top: Montage of a number of frames from a 2D time-lapse image sequence. In this case the sequence was artificially created by our particle track generator developed for controlled quantitative validation of tracking algorithms. Left bottom: Spatiotemporal view of the particle tracks. The track coordinates are known exactly (floating-point precision) at the sampling times and can be interpolated using cubic splines to obtain continuous track representations. Right top: Combined visualization of frames and tracks giving a qualitative impression of the accuracy and consistency of the tracking results. Note that such representations are suitable only for 2D time-lapse data. Visualization of 3D time-lapse image data requires animations or projections. Right bottom: Spatiotemporal view of particle tracks from an artificial 3D time-lapse image sequence (not shown), with the $t$-coordinate indicated along the trajectories by small spheres. Since the spacing between time points is indicative of the velocity of a particle, this may serve as a cue for consistency checking if velocities are known to vary only gradually.

Apart from assisting in the verification of tracking results, such visualizations also give first impressions of possible trends in the data, which may motivate specific quantitative analyses.

Once tracking results are verified (and possibly corrected), a host of motion parameters can be derived from them. Displacements, velocities, and accelerations are easily computed per particle and even per time point or interval. Generally, these values are studied collectively over larger numbers of particles, lumping 
the intrinsic heterogeneity of particle behavior into histograms that reveal the most dominant modes of motion. This also allows the discovery of rare phenomena, which can subsequently be studied in detail by mapping back to the original data. Another frequently studied motion parameter is the mean squared displacement (MSD), which summarizes particle displacement behavior over an entire trajectory. Averaged over subpopulations, it enables the computation of their diffusion coefficients and exponents [5, 12]. An important observation made recently [22] is that particle trajectories should really be studied in 3D over time: 2D data analyses may lead to significant information loss and severe misinterpretation.

With the development of more robust automated tracking methods in cellular and molecular biology it will become more and more possible to accumulate large trajectory databases that allow statistical distinction of behavioral heterogeneity from measurement noise at the single particle level [23]. The analysis of behavioral heterogeneity defines an emerging paradigm in molecular biology, the goal of which is to identify all possible states and the relevant state transitions of a system in its natural mode of action. This approach is likely to reveal the mechanism of cellular homeostasis that underlies robustness in life. Knowing the state space of a healthy molecular process, it will be much easier to understand abnormal behavior that leads to disease and to define strategies that return the deviated system to its normal states. Therefore, comprehensive and automated analysis of large scale experimental data is an urgent item on the biomedical research agenda, placing image analysis and pattern recognition into the center of progress.

\section{PARTICLE TRACKING RECONSIDERED}

Since it must be our aim to minimize manual labor while improving sensitivity and objectivity, and ultimately to replace human beings, in the analysis of cellular and molecular images, it is worth studying how the discussed techniques compare to motion perception in the human visual system. Although less accurate and reproducible in determining the precise coordinates of particles, expert human observers are still much better at confirming the presence or absence of a particle at very low fluorescence levels and making correspondences between frames at relatively high particle densities. It is an experimental fact that even at SNRs as low as $1 \mathrm{~dB}$, in which case signals are virtually indistinguishable from the noise in the individual frames of a time-lapse series, humans are still able to "see" a particle and to make rough estimates of its direction and velocity when shown the series as a movie, at a suitable frame rate. Given the poor performance of current approaches to computerized tracking under more severe practical conditions, one wonders about the qualities of the human visual system that explain its superiority in such cases.

Recent experimental psychophysical and neurological studies have revealed important aspects of human vision that may provide clues. One aspect, especially relevant to larger objects, is that visual motion perception involves multiple motion systems, of which the subsystem of local detectors that responds just to moving luminance patterns is but the lowest-order and simplest. Higher-order systems involve texturesensitive units and differential salience-weighting of features determined by selective attention [24]. Another important finding is that the detection of extended trajectories is mediated by a flexible network of detectors that propagate activation among units tuned to similar directions of motion [25]. Effectively, the network operates as a directionally-selective spatiotemporal integrator: motion detectors selective to all directions of motion at many spatiotemporal scales exist at each location in the visual field. Temporal integration and assumptions about temporal coherence are of crucial importance in detecting local motion of weak and/or ambiguous spatial distributions [26]. Of equal importance are the cognitive processes involved in human visual motion perception. Generally, expert biologists are able to make more sense out of the data than do non-experts, through the use of a priori knowledge about the dynamics.

One of the striking characteristics of most past and current approaches to particle tracking, however, is the rather strict discrimination between spatial and temporal information. Methods for detecting particles 
and estimating their positions per frame typically rely on spatial image features only and do not incorporate information from other frames. Nor do they yield many clues regarding possible interframe correspondences. In turn, methods for addressing the resulting linking problem are generally limited to considering only two or three frames at a time. Alternatively, more integrated optic flow techniques have been proposed [15], which consist in solving a spatiotemporal differential equation for every point in spacetime. While useful for computing velocity fields and distributions, such techniques do not yield explicit particle positions and correspondences without further processing, and they are suitable only in the case of displacements between time frames that are relatively small compared to the size of the object [2]. Finally, the use of prior knowledge to detect weak signals and to resolve ambiguous signals has so far been limited mainly to restricting the allowed maximum particle velocity, or displacement between time frames. These observations suggest interesting directions for future research.

\section{FUTURE CHALLENGES}

Advances in biological imaging technology continue to provide new opportunities in unraveling the complex processes underlying the basic building blocks of life. Molecular biology research has only just begun to study how proteins are spatially and temporally organized in larger functional units and how they behave under the influence of selective perturbations of the system by genetic and molecular interventions. Answering these questions will be critical to understanding diseases and our ability of designing more effective drugs and therapeutic strategies. Since more and more research is being done in living cells, with high-resolution time-lapse image data sets that are not only very large in size but also highly variable and complex, research in this area is rapidly becoming dependent on automated techniques for image processing and analysis. Commercial software packages with modules for particle tracking and motion analysis are already available, but it is highly unlikely that a general-purpose algorithm, developed to provide a solution to many different tracking problems, is going to be the best fit to any particular tracking problem. Furthermore, although there is still room for improving light microscopic imaging (witness the recent development of 4Pi-confocal microscopy and image-interference widefield microscopy) and considerable progress can be expected from new physical concepts capable of breaking the diffraction barrier [27], some of the fundamental imaging problems and limitations mentioned in this article will never be entirely eliminated. As new biological questions continue to be posed and addressed with new imaging technology, there will be a continuing demand for new and improved, dedicated tracking and motion analysis algorithms. Thus the future is bright for researchers in signal and image processing.

From the above considerations, a number of possible research directions emerge as particularly challenging. Studies in human vision seem to indicate that integration is the keyword to developing more robust tracking algorithms. Whereas some have proposed to generate continuous spacetime reconstructions of particle tracks after detection and linking [14], only very few attempts have been made so far to address the tracking problem itself in a more integrated, spatiotemporal fashion [28,29]. Also very important appears to be the possibility to incorporate prior knowledge of dynamic properties, that is of spatiotemporal geometry and topology. This requires research efforts to become increasingly multidisciplinary, with imaging and image processing experts working closely together with biologists in building flexible spatiotemporal models capable of integrating accumulating knowledge about the dynamics of molecular complexes. A different line of research is suggested by the fact that in some studies, images are of such low quality and high complexity that even expert biologists are not able to track individual particles in a deterministic way. More probabilistic approaches to tracking have already been successfully applied in other fields but are relatively new in biological imaging [30]. Finally, we note the importance of developing rigorous validation procedures and standardized protocols to generating test data in order to facilitate objective comparison of different 
algorithms. Ultimately, these efforts will contribute to the verification of biophysical models, leading to a deeper understanding of the molecular mechanisms of life.

\section{ACKNOWLEDGMENTS}

The authors are thankful to Drs. N. Galjart, J. Essers, C. da Silva Almeida, A. Houtsmuller, and G. van Cappellen of the Erasmus MC-University Medical Center Rotterdam, the Netherlands, for fruitful discussions and for providing the image material used in this article. Particle tracking research in the lab of E.M. is funded by the Netherlands Organization for Scientific Research (NWO) through VIDI-grant 639022401, and in the lab of G.D. in parts by NIH grants GM060678, GM067230, and GM068956.

\section{REFERENCES}

Due to space limitations only a subset of papers on the subject could be included. The following list contains mainly recent works and we refer to these and the references therein for more information.

[1] R. Y. Tsien, "Imagining imaging's future”, Nature Cell Biology, vol. 5, pp. S16-S21, September 2003.

[2] D. Gerlich, J. Mattes, and R. Eils, "Quantitative motion analysis and visualization of cellular structures", Methods, vol. 29, no. 1, pp. 3-13, January 2003.

[3] D. J. Stephens and V. J. Allan, "Light microscopy techniques for live cell imaging", Science, vol. 300, no. 5616, pp. 82-86, April 2003.

[4] J. Lippincott-Schwartz and G. H. Patterson, "Development and use of fluorescent protein markers in living cells", Science, vol. 300, no. 5616, pp. 87-91, April 2003.

[5] M. J. Saxton and K. Jacobson, "Single-particle tracking: Applications to membrane dynamics", Annual Review of Biophysics and Biomolecular Structure, vol. 26, pp. 373-399, June 1997.

[6] T. Stepanova, J. Slemmer, C. C. Hoogenraad, G. Lansbergen, B. Dortland, C. I. de Zeeuw, F. Grosveld, G. van Cappellen, A. Akhmanova, and N. Galjart, "Visualization of microtubule growth in cultured neurons via the use of EB3-GFP (endbinding protein 3-green fluorescent protein)", Journal of Neuroscience, vol. 23, no. 7, pp. 2655-2664, April 2003.

[7] J. Essers, A. B. Houtsmuller, L. van Veelen, C. Paulusma, A. L. Nigg, A. Pastink, W. Vermeulen, J. H. Hoeijmakers, and R. Kanaar, "Nuclear dynamics of RAD52 group homologous recombination proteins in response to DNA damage", EMBO Journal, vol. 21, no. 8, pp. 2030-2037, April 2002.

[8] C. Molenaar, K. Wiesmeijer, N. P. Verwoerd, S. Khazen, R. Eils, H. J. Tanke, and R. W. Dirks, "Visualizing telomere dynamics in living mammalian cells using PNA probes", EMBO Journal, vol. 22, no. 24, pp. 6631-6641, December 2003.

[9] P. Farla, R. Hersmus, B. Geverts, P. O. Mari, A. Nigg, H. J. Dubbink, J. Trapman, and A. B. Houtsmuller, "The androgen receptor ligand-binding domain stabilizes DNA binding in living cells", Journal of Structural Biology, vol. 147, no. 1, pp. 50-61, July 2004.

[10] M. Gu, Advanced Optical Imaging Theory, Springer-Verlag, Berlin, 2000.

[11] M. K. Cheezum, W. F. Walker, and W. H. Guilford, "Quantitative comparison of algorithms for tracking single fluorescent particles", Biophysical Journal, vol. 81, no. 4, pp. 2378-2388, October 2001.

[12] D. S. Martin, M. B. Forstner, and J. A. Käs, "Apparent subdiffusion inherent to single particle tracking”, Biophysical Journal, vol. 83, no. 4, pp. 2109-2117, October 2002.

[13] H. Bornfleth, P. Edelmann, D. Zink, T. Cremer, and C. Cremer, "Quantitative motion analysis of subchromosomal foci in living cells using four-dimensional microscopy”, Biophysical Journal, vol. 77, no. 5, pp. 2871-2886, November 1999.

[14] W. Tvaruskó, M. Bentele, T. Misteli, R. Rudolf, C. Kaether, D. L. Spector, H. H. Gerdes, and R. Eils, "Time-resolved analysis and visualization of dynamic processes in living cells", Proceedings of the National Academy of Sciences of the United States of America, vol. 96, no. 14, pp. 7950-7955, July 1999.

[15] D. Uttenweiler, C. Weber, B. Jähne, R. H. A. Fink, and H. Scharr, "Spatiotemporal anisotropic diffusion filtering to improve signal-to-noise ratios and object restoration in fluorescence microscopic image sequences", Journal of Biomedical Optics, vol. 8, no. 1, pp. 40-47, January 2003. 
[16] C. Ó. S. Sorzano, P. Thévenaz, and M. Unser, "Elastic registration of biological images using vector-spline regularization", IEEE Transactions on Biomedical Engineering, vol. 52, no. 4, pp. 652-663, April 2005.

[17] B. Rieger, C. Molenaar, R. W. Dirks, and L. J. van Vliet, "Alignment of the cell nucleus from labeled proteins only for 4D in vivo imaging", Microscopy Research and Technique, vol. 64, no. 2, pp. 142-150, June 2004.

[18] R. J. Ober, S. Ram, and E. S. Ward, "Localization accuracy in single-molecule microscopy", Biophysical Journal, vol. 86, no. 2, pp. 1185-1200, February 2004.

[19] D. Thomann, D. R. Rines, P. K. Sorger, and G. Danuser, "Automatic fluorescent tag detection in 3D with super-resolution: Application to the analysis of chromosome movement", Journal of Microscopy, vol. 208, no. 1, pp. 49-64, October 2002.

[20] J.-C. Olivo-Marin, "Extraction of spots in biological images using multiscale products", Pattern Recognition, vol. 35, no. 9, pp. 1989-1996, September 2002.

[21] C. J. Veenman, M. J. T. Reinders, and E. Backer, "Resolving motion correspondence for densely moving points", IEEE Transactions on Pattern Analysis and Machine Intelligence, vol. 23, no. 1, pp. 54-72, January 2001.

[22] J. F. Dorn, K. Jaqaman, D. R. Rines, G. S. Jelson, P. K. Sorger, and G. Danuser, "Yeast kinetochore microtubule dynamics analyzed by high-resolution three-dimensional microscopy”, Biophysical Journal, vol. 89, no. 4, pp. 2835-2854, October 2005.

[23] F. S. Soo and J. A. Theriot, "Large-scale quantitative analysis of sources of variation in the actin polymerization-based movement of listeria monocytogenes”, Biophysical Journal, vol. 89, no. 1, pp. 703-723, July 2005.

[24] Z.-L. Lu and G. Sperling, "Three-systems theory of human visual motion perception: Review and update", Journal of the Optical Society of America A: Optics, Image Science, and Vision, vol. 18, no. 9, pp. 2331-2370, September 2001.

[25] P. Verghese, S. N. J. Watamaniuk, S. P. McKee, and N. M. Grzywacz, "Local motion detectors cannot account for the detectability of an extended trajectory in noise", Vision Research, vol. 39, no. 1, pp. 19-30, January 1999.

[26] P.-Y. Burgi, A. L. Yuille, and N. M. Grzywacz, "Probabilistic motion estimation based on temporal coherence", Neural Computing, vol. 12, no. 8, pp. 1839-1867, August 2000.

[27] S. W. Hell, M. Dyba, and S. Jakobs, "Concepts for nanoscale resolution in fluorescence microscopy", Current Opinion in Neurobiology, vol. 14, no. 5, pp. 599-609, October 2004.

[28] D. Sage, F. R. Neumann, F. Hediger, S. M. Gasser, and M. Unser, "Automatic tracking of individual fluorescence particles: Application to the study of chromosome dynamics", IEEE Transactions on Image Processing, vol. 14, no. 9, pp. 1372-1383, September 2005.

[29] S. Bonneau, M. Dahan, and L. D. Cohen, "Single quantum dot tracking based on perceptual grouping using minimal paths in a spatiotemporal volume", IEEE Transactions on Image Processing, vol. 14, no. 9, pp. 1384-1395, September 2005.

[30] A. Genovesio, B. Zhang, and J.-C. Olivo-Marin, "Interacting multiple model based method to track moving fluorescent biological spots", in IEEE International Symposium on Biomedical Imaging: Macro to Nano, R. Leahy and C. Roux (eds.), vol. 2, IEEE, Piscataway, NJ, pp. 1239-1242, April 2004. 